Case Report

\title{
Microcystic Serous Cystadenoma of Pancreas Presenting With Chronic Diarrhea
}

\author{
Shravya Sudesh Kotian ${ }^{1}$, Shubha P Bhat', Rajshekhar M ohan ${ }^{3}$, Sajitha K ${ }^{4}$ \\ ${ }^{1}$ Resident, ${ }^{2}$ Associate Professor, ${ }^{4}$ Assistant Professor Department of Pathology, ${ }^{3}$ Professor \& Head, Department of Surgery, \\ K.S Hegde M edical Academy, Nitte-Deemed to be University, M angalore, Karnataka, India. \\ *Corresponding Author : Shubha P Bhat, Associate Professor, Department of Pathology, K SHegde M edical Academy, Nitte- Deemed to \\ beUniversity, Mangalore, Karnataka, India- 575 018, M obile : +919986136125, E-mail : bhatshubha_257@ rediffmail.com
}

$\begin{array}{ll}\text { Received } & : 25.01 .2018 \\ \text { Review Completed } & : 20.05 .2018 \\ \text { Accepted } & : 15.07 .2018\end{array}$

Keywords : Pancreas; Serous cystadenoma; Whipplessurgery

\begin{tabular}{|c|}
\hline Access this article online \\
\hline Quick Response Code \\
\hline \\
\end{tabular}

\begin{abstract}
Pancreatic cystic neoplasms are increasingly being recognized due to widespread use of advanced imaging techniques. The prevalence of pancreatic cystic lesions is estimated to be between $2.6 \%$ - 19.6\%. Serous Cystic Neoplasms account for nearly 16\% of surgically resected pancreatic cystic neoplasms. They commonly occur in females between $5^{\text {th }}$ and $6^{\text {th }}$ decade of life. These tumours are essentially benign and asymptomatic unless they attain a large size. Majority of them occur in the body and tail of pancreas. M alignant transformation is reported in $1 \%-3 \%$ of cases. Surgical excision is the treatment of choice. Thus, it is important to accurately diagnose this lesion to avoid unnecessary intervention in the form of chemotherapy or uncalled for surgical resection. We report a rare case of a 65 year old male who presented with a history of intermittent diarrhea.
\end{abstract}

\section{Introduction}

Pancreatic cystic neoplasms (PCNs) are increasingly being recognized incidentally with widespread use of advanced imaging techniques (1). The prevalence of pancreatic cystic lesions on abdominal imaging has been reported to be between $2.6 \%$ - 19.6\%. PCNs comprise serous cystic neoplasms (SCNs), mucinous cystic neoplasms(MCNs), intraductal papillary mucinous neoplasms(IPMNs) and solid pseudopapillary neoplasms (SPPNs). SCNsaccount for nearly $16 \%$ of surgically resected PCNs and $>30 \%$ of all clinically diagnosed PCNs. Most serous cystic neoplasms are benign and represent pancreatic serous cystadenomas (SCAs) $(2,3)$.They occur most frequently in females and occur in the age group between 56 and 62 years. SCA can occur in any region of the pancreas. Approximately $60 \%$ arise from the pancreatic body and tail and $40 \%$ arise from the head of the pancreas and uncinate process. If the mass occurs in the pancreatic head, it can result in gastrointestinal or biliary tract obstruction, and when sufficient islet cell tissue is destroyed by the tumour, diabetes mellitus may also occur $(2,4)$.Up to $60 \%$ of patients are asymptomatic. Alternatively, patients may present with nonspecific symptoms such as abdominal distension, abdominal pain and rarely jaundice. Computed tomography (CT), Magnetic resonance imaging (M RI) and endoscopic ultrasound (EUS) are the three most commonly used imaging techniques for revealing SCNs.

Surgical excision is the treatment of choice for large tumors. Whipples procedure, Begars procedure, spleenpreserving distal pancreatectomy or central segmental pancreatectomy are the surgical treatments based on the location of the tumour(1).

Histopathology shows varying sized cystic spaces lined by glycogen rich epithelial cells typically without mitosis. Centrally located fibrous tissue ( scar) can also be found (5). Here we report a case of 65 year old male presenting with intermittent loose stools since 2 years with increasing 
frequency since 2 months. Radiological evaluation showed a well circumscribed heterogenous enhancing mass in head and uncinate process of pancreas. Whipples procedure was done and histopathology confirmed the diagnosis of microcystic serous cystadenoma. There was also an anomalous low insertion of cystic duct into right hepatic duct.

\section{Case Report}

A 65 year old presented to the department of general surgery with complaints of passage of intermittent loose stools for 2 years which aggravated since 2 weeks. Daily there were 3-4 episodes of loose stools, 10-15 mins after food intake. Stools were watery in consistency and there was no associated passage of mucous or blood. Patient gave a history of loss of appetite.There was no history of abdominal pain, mass per abdomen or incontinence. The patient had no associated family history or other comorbid conditions. CT scan revealed a heterogeneously enhancing multi-cystic lesion in the head and uncinate process of pancreas. A provisional diagnosis of side branch intraductal papillary mucinous neoplasms (IPMN) or serous cystadenoma was made. Hematological investigations, renal function tests and liver function tests were within normal limits; LDH (Lactate Dehyrdrogenase) level was 193 $\mathrm{mg} / \mathrm{dl}$; CEA (Carcinoembryonic Antigen) level was 2.87 $\mathrm{ng} / \mathrm{ml}$; Serum Amylase levels were 79 U/L (20-160 U/L).

Whipples resection was done which included, pancreaticoduodenectomy and cholecystectomy specimen with attached cystic duct and right and left hepatic duct. The pancreatico-duodenectomy specimen showed a well circum scribed tumor measuring $5 \mathrm{~cm} \times 6 \mathrm{~cm} \times 4 \mathrm{~cm}$ arising from the head and uncinate process of the pancreas. Central scar was also noted. The cut surface of the tumor had a microcystic, spongy appearance and exuded serous fluid. Adjacent pancreatic tissue appeared normal (figure 1). An anomalous low insertion of the cystic duct with right hepatic duct was seen. Gall bladder appeared to be dilated and distended. On cut surface, bile was present and mucosa appeared granular.

Histopathological examination revealed a tumor containing varying sized dilated cystic spaces lined by cuboidal to flattened epithelial cells (figure 2). The cells had round to oval uniform nucleus, fine chromatin and inconspicuous nucleoli. Cytoplasm was clear to eosinophilic (figure 3). At places stratification and papillary projection were seen into the lumen. Lumen showed eosinophilic secretions and at places haemorrhage. The cysts were separated by delicate fibrous septa containing lymphocytes, plasma cells and occasional eosinophils. Adjoining pancreatic tissue and pancreatic ducts, gall bladder had unremarkable findings.

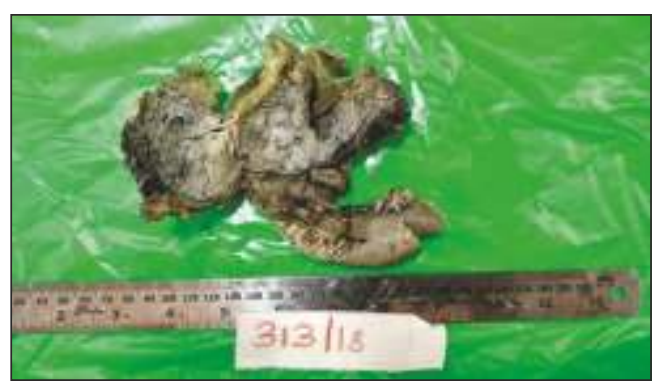

Figure 1 : Pancreatico-duodenectomy specimen, Cut surface of pancreas shows a well circumscribed tumor containing numerous small cystic spaces.

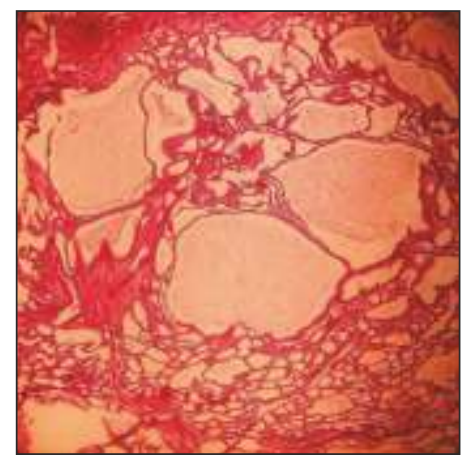

Figure 2 : Tumor shows numerous cystic spaces. Haematoxylin and Eosin Stain. 10x

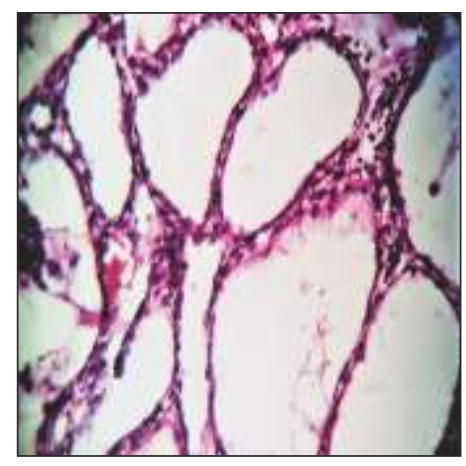

Figure 3 : The cystic spaces are lined by cuboidal cells with round to oval nucleus with inconspicuous nucleoli and contain clear to eosino philic cyto plasm. Haematoxylin and Eosin stain ,40x. 
Based on these findings a diagnosis of M icrocystic serous cystadenoma of pancreas with anomalous low insertion of cystic duct into right hepatic duct was made.

\section{Discussion}

Serous cystadenoma of the pancreas was first described by Compagno and Oertel in 1978 (3). The prevalence of pancreatic cystic lesions on abdominal imaging has been reported to be between $2.6 \%-19.6 \%$. SCNs account for nearly $16 \%$ of surgically resected PCNs and $>30 \%$ of all clinically diagnosed PCNs. Most serous cystic neoplasms are benign and represent pancreatic serous cystadenomas (SCAs). They occur more commonly in females and present between 56 years- 62 years of age $(2,3)$. Up to $60 \%$ of patients are asymptomatic, although larger tumors may present with nonspecific symptoms such as abdominal pain, abdominal mass and even jaundice. If the mass occurs in the pancreatic head, it can result in gastrointestinal or biliary tract obstruction, and when sufficient islet cell tissue is destroyed by the tumour, diabetes mellitus may also occur. In our case, patient was a 65 -year-old male who presented with complains of intermittent loose stool since 2 years which aggravated since 2 months.

Ultrasound (USG), CT, MRI and EUS are the imaging techniques employed to detect $\mathrm{SCN}$ s. CT remains the first line imaging modality due to more widespread availability. EUS has the ability to collect fluid from cystic lesions via fine-needle aspiration (FNA) for cytological and biochemical analysis, such as carcinoembryonic antigen (CEA), amylase, and KRAS mutations. Radiologically, pancreatic serous cystadenomas have been described as multi-loculated cystic masses with central stellate scars and calcifications.

Following are the various patterns of pancreatic serous cystadenoma.

M ICROCYSTIC PATTERN-The microcystic pattern is present in $70 \%$ cases of pancreatic serous cystadenomas. They are characterized by a polycystic pattern of multiple cysts separated by fibrous septa that can coalesce to form a central scar. These cysts measure $2 \mathrm{~cm}$ or smaller (usually more than 6). Fine external lobulations is a common and characteristic feature (6).

HONEYCOM B PATTERN-The honeycomb pattern is seen in approximately $20 \%$ of patients is characterized by numerous sub centimetre cysts that cannot be individually distinguished bycross-sectional imaging.

OLIGOCYSTIC (I.E. MACROCYSTIC) PATTERN-The oligocystic pattern is seen in fewer than $10 \%$ of patients. This pattern exhibits large cysts measuring $>2 \mathrm{~cm}$.

SOLID PATTERN-Solid pattern of serous cystadenoma, is a rare variant where the cells are arranged in nests, sheets and trabeculae.

Most of the tumours exhibit in negligible growth. Those lesions that do grow usually grow slowly ( $\varangle \mathrm{mm} /$ year) $(6$, 7).

In our patient,CT scan revealed a well-defined heterogeneously enhancing mixed density lesion located in the head and uncinate process located in the pancreas. Multiple non-enhancing hypodense cystic areas with thick and thin septations were noted. It appeared to abut the anterior wall of the second, and third part of duodenum.Provisional diagnosis of side branch IPMN or serous cystadenoma was made.

A CEA level of $<5 \mathrm{ng} / \mathrm{mL}$ is $95 \%$ specific for $\mathrm{SCN}$, pseudocyst or primary neuroendocrine tumor ( $\mathrm{pNET}$ ). An elevated CEA level favours a mucinous lesion. CEA threshold varies in different centre's, ranging from $5 \mathrm{ng} / \mathrm{mL}$ to $>100 \mathrm{ng} / \mathrm{ml}$. An amylase level $<250 \mathrm{U} / \mathrm{L}$ also favours diagnosis of SCN. Our patient had CEA and Amylase levels of $2.87 \mathrm{ng} / \mathrm{ml}(0-4$ $\mathrm{ng} / \mathrm{ml}$ )and $79 \mathrm{U} / \mathrm{L}(20-160 \mathrm{U} / \mathrm{L})$ respectively. Other than CEA and amylase, levels of cystic fluid metabolites, glucose and kynurenine are markedly elevated in SCNs compared to M CNs. This aids the diagnosis of SCNs $(1,2,6)$.

Currently, there is no universal consensus on the best management strategy, but it is widely accepted that not every single case should be surgically resected. Resection should be considered for large $(>4 \mathrm{~cm})$, rapidly growing 
SCNs, given that such SCNs are more likely to cause symptoms. Indications for surgical intervention include presence of symptoms or an uncertain diagnosis.

Surgical resection of an SCN largely depends on the location of the lesion. Because of the benign nature of SCN, it is recommended that pancreatic functions are protected and preserved as much as possible for better outcome and quality of life. If SCNs are localized in the pancreatic head, pylorus-preserving pancreatoduodenectomy or Begar procedure is often carried out. If $\mathrm{SCN}$ s are located in the body or tail of the pancreas, spleen-preserving distal pancreatectomy should be the first choice. For patients whose SCN sare located in the neck of the pancreas, central segmental pancreatectomy is an alternative procedure, preserving islet cell mass and reducing the risk of iatrogenic insulin dependent diabetes. Enucleation is not recommended because greater morbidity (up to $35 \%$ ) and associated complications such as pancreatic fistula have been reported (1).Our patient underwent a Whipples procedure and a Pancreaticoduodenectomy specimen along with cholecystectomy specimen with attached cystic duct and hepatic ducts were sent to the histopathology laboratory for confirmation of diagnosis.

Gross appearance of a serous cystadenoma is that of a lobulated mass comprising of numerous cystic spaces and usually measuring $2 \mathrm{~cm}$ or smaller.These cysts produce watery fluid. Often a central scar is also noted.The tumor has a spongy appearance. Histopathology shows glycogen rich epithelial cells that form innumerable small thin walled cysts containing serous fluid.

In our experience the Whipples resection specimen showed a well circumscribed tumor measuring $5 \mathrm{~cm} \times 6 \mathrm{~cm} \times$ $4 \mathrm{~cm}$ arising from the head and uncinate process of the pancreas.. Central scar was also noted. The tumor had a microcystic spongy appearance and exuded serous fluid. Adjoining pancreatic tissue appeared normal.An anomalous insertion of the Cystic duct with right hepatic duct at lower level was seen.

On Microscopy the tumour showed varying sized dilated cystic spaces lined by cuboidal to flattened epithelial cells. The cells had round to oval uniform nucleus, fine chromatin and inconspicuous nucleoli,at places stratification and papillary projection were seen into the lumen. Lumen showed eosinophilic secretions and at places haemorrhage. The cysts were separated by delicate fibrous septa containing lymphocytes, plasma cells and occasional eosinophils. Adjoining pancreatic tissue was normal. Based on these findings a final diagnosis of Serous Microcystic cystadenoma of head of Pancreas with low insertion of cystic duct into right hepatic duct was made.

Serous cystadenomas generally have a good prognosis (8).According to WHO the prevalence of malignancy of serous cystic neoplasms of the pancreas is $1 \%-3 \%$. To date serous cystadenoma is considered malignant when tumor invasion of surrounding tissues and organs or distant metastasis are present (9). Follow up strategies have not been standardized for serous cystic neoplasms. Some advocate follow up imaging every 12 months, while others suggest biennial surveillance. Our patient is on follow up since sixmonths and is doing well.

\section{Conclusion}

SCNs are uncommon benign tumors that are more usually asymptomatic and found incidentally, although symptoms may be produced when the tumor attains a large size. CT and MRI are the most commonly used methods to diagnose SCA. The most common variant is the microcystic pattern, characterized by multiple cysts measuring $2 \mathrm{~cm}$ or smaller. Fine external lobulations is a common and characteristic feature. On microscopic examination, these cysts are lined by cuboidal cells and separated by fibrous septa. Surgical management is advised only for those lesions which measure more than $4 \mathrm{~cm}$ and produce symptoms. Surgical intervention can vary depending on the site of the tumor. Enucleation however is never recommended.A multi-disciplinary approach combining newer detection methods like CEA, Amylase and cytokeratins should be employed for future management and research. 


\section{References}

1. Zhang X-P, Yu Z-X, Zhao Y-P, Dai M-H. Current perspectives on pancreatic serous cystic neoplasms: diagnosis, management and beyond. World J Gastrointest Surg. 2016 ;8(3):202-11.

2. Chu LC, Singhi AD, Haroun RR, Hruban RH, Fishman EK. The many faces of pancreatic serous cystadenoma: radiologic and pathologic correlation. Diagnostic and interventional imaging. 2017 ;98(3):191202.

3. Gao Y, Zhang JP, Zhu P. Serous cystadenoma of the pancreas with atypical clinical manifestations: A case report. Molecular and clinical oncology. 2016;4(4):584-6.

4. Üstün M Ö, Tugyan N, Tunakan M. Coexistence of an endocrine tumour in a serous cystadenoma (microcystic adenoma) of the pancreas, an unusual association. Journal of clinical pathology. 2000 Oct 1;53(10):800-2.

5. Dietrich $C F$, Dong $Y$, Jenssen $C$, Ciaravino V, Hocke $M$, Wang WP, Burmester E, M oeller K, Atkinson NS, Capelli P, D'Onofrio M. Serous pancreatic neoplasia, data and review. World journal of gastroenterology. 2017; 23(30):5567.

6. Choi JY, Kim MJ, Lee JY, Lim JS, Chung JJ, Kim KW, Yoo HS. Typical and atypical manifestations of serous cystadenoma of the pancreas: imaging findings with pathologic correlation. American Journal of Roentgenology. $2009 ; 193(1): 136-42$.

7. Menard A, Tomlinson G, Cleary S, Wei A, Gallinger S, Haider MA. Serous cystadenomas of the pancreas: long-term follow-up measurement of growth rate. Canadian Association of Radiologists Journal. $2011 ; 62(3): 190-6$.

8. Cicy PJ, Gopinathan J, Ulahannan SE. Morphologic features in cystic lesions of pancreas-a retrospective analysis. International Journal of Advances in Medicine. 2018;5(1):192-6.

9. Bramis K, Petrou A, Papalambros A, M anzelli A, M antonakis E, Brennan $N$, Felekouras E. Serous cystadenocarcinoma of the pancreas: report of a case and management reflections. World journal of surgical oncology. 2012;10(1):51. 